\title{
Minimal Barriers to Invasion During Human Colorectal Tumor Growth
}

Marc D. Ryser ${ }^{1,2,3}$, Diego Mallo ${ }^{4}$ Allison Hall ${ }^{5}$, Timothy Hardman ${ }^{6}$, Lorraine M. King ${ }^{6}$, Inmaculada C. Sorribes ${ }^{2}$, Carlo C. Maley ${ }^{4}$, Jeffrey R. Marks ${ }^{3,6}$, E. Shelley Hwang ${ }^{3,6}$, Darryl Shibata ${ }^{7^{*}}$

${ }^{1}$ Department of Population Health Sciences, Duke University Medical Center, Durham, NC

${ }^{2}$ Department of Mathematics, Duke University, Durham, NC

${ }^{3}$ Duke Cancer Institute, Durham, NC

${ }^{4}$ Arizona Cancer Evolution Center and School of Life Sciences, Arizona State University, Tempe, AZ

${ }^{5}$ Department of Pathology, Duke University Medical Center, Durham, NC

${ }^{6}$ Department of Surgery, Duke University Medical Center, Durham, NC

${ }^{7}$ Department of Pathology, University of Southern California Keck School of Medicine, Los Angeles, CA

*For correspondence: Darryl Shibata, University of Southern California Keck School of Medicine, 1441 Eastlake Avenue, NOR2424, Los Angeles, CA 90033, USA. Email: dshibata@usc.edu 


\section{ABSTRACT}

Intra-tumoral heterogeneity (ITH) could represent clonal evolution where subclones with greater fitness confer more malignant phenotypes and invasion constitutes an evolutionary bottleneck. Alternatively, ITH could represent branching evolution with invasion of multiple subclones. The two models respectively predict a hierarchy of subclones arranged by phenotype, or multiple subclones with shared phenotypes. We delineated these modes of invasion by merging ancestral, topographic, and phenotypic information from 12 human colorectal tumors (11 carcinomas, 1 adenoma) obtained through saturation microdissection of 325 small tumor regions. The majority of subclones $(29 / 46,60 \%)$ shared superficial and invasive phenotypes. Of 11 carcinomas,

9 showed evidence of multiclonal invasion, and invasive and metastatic subclones arose early along the ancestral trees. Early multiclonal invasion in the majority of these tumors indicates the expansion of co-evolving subclones with similar malignant potential in absence of late bottlenecks, and suggests that barriers to invasion are minimal during colorectal cancer growth. 


\section{INTRODUCTION}

Direct observation of the clonal evolutionary process in human tumors is difficult. However, intratumor heterogeneity (ITH) is common in human tumors and the ancestral information recorded by subclonal mutations can be used to reconstruct their growth ${ }^{1-3}$. The interpretation of the ancestral information encoded by ITH is critical because knowledge about tumor initiation and progression informs effective strategies to prevent, detect, and treat human cancers ${ }^{4-6}$.

Previous studies of ITH focused primarily on multiregional bulk sampling of tumors. While this approach enables mapping of ITH during lateral growth, it lacks the spatial and phenotypic resolution needed to characterize ITH during the critical downward growth (invasion) that confers tumor stage and prognosis. In this study, we show that phenotypic, ancestral and topographic information can be merged after saturation microdissection and deep resequencing of tumor sections to characterize the phylogeography of invasion. By localizing subclones directly onto microscope sections we are able to infer the ancestry of each tumor region and the final histologic phenotypes of the subclones.

The path from start of superficial tumor growth to invasion of deep tissue is generally thought to follow a multistep progression ${ }^{7}$. In this model, increasingly fit subclones expand in the superficial layers until an evolutionary bottleneck event gives rise to a subclone that penetrates the muscularis mucosae and invades the deeper tissue (Fig. 1a, left). Consequently, the subclone topography on microscope slides is expected to consist of contiguous subclones layered horizontally by phenotype (Fig. 1b, left), with a single late subclone acquiring the ability to invade the deep tissue (Fig. 1c, left).

Here, we show that the mutation topographies on microscope slides in 12 human colorectal tumors do not support an evolutionary bottleneck model of invasion. To the contrary, we find that the majority of tumors show evidence of multiclonal invasion (Fig. 1a, right), where multiple jjigsaw arrayed subclones span from superficial to invasion regions (Fig. 1b, right). Through reconstruction of ancestral trees we find that invading subclones arise early during growth, suggesting growth dynamics that are expected under branching evolution ${ }^{1,8}$ (Fig. 1c, right) as opposed to a multi-step progression with late invasion (Fig. 1c, left). In particular, the observed phylogeographies are consistent with the neutral Big Bang model of tumorigenesis, a type of branching evolution that has been extensively documented in these tumors. ${ }^{9-11}$ 
In summary, and similarly to a recent study in early stage breast cancers ${ }^{12}$, our results indicate that multiclonal invasion is common in colorectal tumors and indicate that there are only minimal barriers to invasion after the start of tumor growth.

\section{RESULTS}

Saturation microdissection of tumor slides. A prerequisite for mapping the topography of tumor subclones is the ability to physically isolate regions small enough to contain a single subclone. Fortunately, prior studies indicate that single colorectal tumor glands are defined subclones because they are clonal for the public (found in all sampled regions) and private (found in a strict subset of sampled regions) mutations identified through exome sequencing of bulk samples ${ }^{9}$. To leverage this gland level clonality, we microdissected individual spots (small regions consisting of 2-5 adjacent glands each) from multiple regions of microscope slides (Fig 2a) obtained from 12 human colorectal tumors (Table 1). From each primary tumor, two (one for tumor R) arbitrarily located sections were collected and a median of 13 spots (range: 6-20) per slide were microdissected. The average minimum distance between neighboring spots was $2.9 \mathrm{~mm}$, with an average pairwise distance of 8.4 $\mathrm{mm}$ between dots on the same slide. Additional sections from metastatic sites were obtained and microdissected for three tumors $(\mathrm{J}, \mathrm{C}, \mathrm{H})$.

Distinct subclones are present in small tumor regions. For each tumor, a panel of candidate mutations (median number of loci: 47, range: $13-110)$ was derived from a previous whole exome sequencing study ${ }^{9}$ (Fig. $^{2}$ 2b). Deep (mean depth $\sim 9,000 x$; minimum depth: 20x) targeted re-sequencing of the tumor-specific mutation panels was performed. Variant allele frequencies (VAF) of private mutations were comparable to those of public mutations (Fig. S1), consistent with the presence of a single subclone in each spot. Indeed, of 325 analyzed spots, only 4 harbored multiple subclones as indicated by low private mutation VAFs and homoplasy (Table S1). The remaining 321 clonal spots were assigned a genotype, enabling the mapping of subclone topographies (Figs 2c, 3, 4, S2: panel i-ii) and reconstruction of maximum parsimony phylogenies (Figs 2c, 3, 4 and S2: panel iii; Fig. 4: panel iv). The jigsaw-arranged subclones varied in their topographic spread, with a median of 3.5 spots 
per subclone (range: 1 to 27; Fig. 5a). There was a median of 3 subclones per slide (range: 1-7; Fig. 5b) and a median of 5 subclones per tumor (range: 1 to 9; Fig. 5c).

Most subclones share superficial and invasive phenotypes. The merged ancestral, topographic and phenotypic information enabled us to test whether observed ITH was more consistent with late bottleneck or multiclonal invasion (Fig. 1). To this end, we first combined histopathologic examination and spatial registration to classify each microdissected spot as either superficial (glandular mucosa above the muscularis mucosae), invasive (below the muscularis mucosae) or metastatic. We found that subclones with multiple spots were commonly arrayed in contiguous vertical columns spanning from superficial to invasive regions (Figs. 2c, 3, 4, S2: panel ii). Across 10 carcinomas with at least one invasive spot in the primary tumor (thus excluding tumor C), the majority of subclones (29/46, or $63 \%$ ) had a mixed phenotype, that is they covered both superficial and invasive regions (Fig. 5d). Further restricting the analysis to the 37 subclones with more than one spot, the fraction of mixed subclones increased to $76 \%$. Subclone phenotypic heterogeneity is visualized on t-SNE plots where genotypes but not phenotypes cluster together (Figs 5e, S3). In summary, instead of subclones layered horizontally by phenotype (Fig. 1b, left) as predicted by multistep progression, the majority of subclones were of mixed phenotype (Fig. 1b, right).

Fingerprints of multiclonal invasion. Next, we sought to reconstruct the invasion dynamics in the 11 invasive carcinomas. Among 6 of these, invading subclones (of either invasive or mixed phenotype) formed a polyphyletic group in the ancestral tree, indicating multiclonal invasion (Table 1; Figs. 2c, 3, s2: panel iii; Fig. 4: panel iv). Further assuming that once invasive, subclones did not migrate back across the muscularis mucosae into superficial regions, we were able to reconstruct the number of invasion events for each tumor (Figs 2c, 3, S2: panel iv; Fig. 4: panel v). Of the 10 polyclonal CRCs (tumor $\mathrm{J}$ was monoclonal), only tumor $\mathrm{C}$ had a single invasion event compatible with an evolutionary bottleneck. The remaining 9 carcinomas had undergone multiclonal invasion, with a median of 3 invasion events per tumor (range: 1-7; Table 1). In summary, evidence of multiclonal invasion in 9 of 11 carcinomas suggests that the observed ITH is consistent with a branching model of evolution that gives rise to multiple invading subclones during growth (Fig. 1c, right).

Similar growth and invasion dynamics for early and late ancestral subclones. We examined whether late branching subclones may experience stepwise increases in fitness and thus more advanced phenotypes. To 
this end, we subdivided subclones into "early" or "late" categories based on whether they arose during the first or second half of tumorigenesis, as measured by the distance from the root in the maximum parsimony trees (Fig. S4c). Among carcinomas, 21 and 34 subclones were found on early and late branches, respectively. Early invasion events were found in all 11 CRCs (Table 1), and there was no size difference between early and late subclones (Wilcoxon rank-sum test: $p=0.7$ ), which both had a median size of 4 spots per clone (Fig. $5 f$ ). In summary, there was no evidence that later subclones were phenotypically more advanced than earlier subclones.

Jigsaw subclone topography. Because tumors grow by gland splitting or fission ${ }^{13}$, neighboring glands should be related and subclones expand continuously. Consistent with these predictions, most subclones with more than 2 spots in a slide were spatially contiguous (41 of 46 , or $89 \%$ ). Although contiguous subclones are expected with both stepwise progression and single neutral expansions, the phylogenetic relatedness of neighboring subclones can provide further delineation between the models. We found that the normalized genetic distance between adjacent subclones had a wide distribution (Fig. $5 \mathrm{~g}$ ), suggesting that ancestry did not dictate subclone topography as would be expected in stepwise progression. In addition, correlations between spatial and genetic distances of spots within tumors were low, with a median Pearson correlation of 0.37 (Fig. 5h). This indicates that the co-evolution of equivalent subclones may have been preceded by early subclone mixing ${ }^{9}$, leading to the observed jigsaw arrangements of subclones in the final tumor. In summary, the observed subclone topographies are consistent with punctuated evolution followed by neutrally expanding subclones (Fig. 1c, right), as posited by the Big Bang theory of colorectal carcinogenesis. ${ }^{11}$

Recognizing stepwise progression. Although a lack of clear morphologic boundaries or size differences between the jigsaw arrayed subclones is consistent with tumorigenesis by near-neutral subclones, the presence of clonal selection is difficult to rule out ${ }^{14,15}$. Indeed, a key challenge in recognizing selection during tumor growth is that the relative fitness of a subclone needs to be very high in order to "outrun" its neighboring, neutrally expanding subclones ${ }^{9,16,17}$. Focusing instead on established canonical driver mutations, we found that 11 of 12 tumors had at least one public driver mutation (tumor $\mathrm{H}$ had none). Two tumors ( $\mathrm{D}$ and $\mathrm{E})$ had additional private driver mutations that may have conferred an increase in fitness to individual subclones during expansion. In tumor D (Fig. 3a), the largest subclone had its own private TP53 mutation (C238Y) and had the histologic 
features of stepwise progression with central invasion and relatively distinct morphologic boundaries. However, this subclone did not arise in stepwise fashion from the surrounding superficial subclones as indicated by the presence of two superficial subclones that arose later in the ancestral tree and harbored their own private TP53 mutations (V143A, R248Q). In tumor E (Fig. 3b), both early branches had their own private driver mutations (APC and PIK3CA, and APC). In summary, in the analyzed tumors we found only limited evidence of selection through the acquisition of private driver mutations.

Invasion and metastasis can occur very early. Finally, we looked for direct evidence of stepwise progression by examining areas with early phenotypic transition; that is situations where a small number of cells change the phenotypic classification of the tumor. A critical criterion for identifying early malignant progression is whether tumor cells have invaded the stalk of an adenoma. The sole adenoma in this study $(\mathrm{K})$ was large $(6 \mathrm{~cm})$ and only few cells had microscopically invaded the stalk (Fig 3c). In the stepwise adenoma-cancer sequence, this early invasion is expected to occur right after a single adenoma subclone accumulates a driver mutation necessary for invasion. However, of the three subclones with stalk invasion, two arose very early in the tree (Fig. 3c,iii). Another example of early transition is the microscopic presence of small metastatic foci which upgrade clinical staging, confer poorer prognosis, and represent the zenith of stepwise progression. We analyzed three CRCs $(\mathrm{J}, \mathrm{C}, \mathrm{H})$ with small microscopic metastases in the lymph nodes and liver (H only) (Fig. 4). In all cases the metastatic spots were genotypically identical within a patient and lacked any of the private mutations of the primary, indicating early divergence. In two cases (Fig. 4a-b), the microscopic metastatic foci had all the public mutations; in tumor $\mathrm{H}$ (Fig. 4c), the microscopic metastases diverged even earlier as evidenced by the lack of 10 of 22 public mutations present in the primary tumor. Of note, none of the canonical CRC drivers were missing in the metastatic subclones. In summary, in these four examples the acquisition of more advanced phenotypes occurred along the earliest ancestral branches, indicating that invasion and metastasis may occur very early during growth.

\section{DISCUSSION}

Tumors are heterogenous populations of cells with different phenotypes and multiregional sampling has revealed that genetic ITH is also very common. However, there is uncertainty about the number and size of subclones, 
how they are arranged spatially, and whether subclones with different genotypes have distinct phenotypes. Here we addressed these questions through saturation microdissection and targeted resequencing of tumor sections on microscope slides, assessing the spatial distributions of subclones and their histologic phenotypes.

Consistent with the classical model of stepwise progression, colon cancers have a hierarchy of progressing phenotypes, from superficial to deeply invasive cells. This downwards spatial histologic progression also corresponds to clinical staging criteria, where more advanced cancers of poorer prognosis are defined by more deeply invasive or metastatic tumor cells. Similar to the phenotypic hierarchy, genetic ITH can be cast as a hierarchy of early and late branching subclones along the phylogenetic tree. Under stepwise progression, the phylogenetic hierarchy is expected to directly reflect histologic barriers to progression (bottlenecks). More precisely, early subclones with limited malignant capabilities can spread horizontally, but not downward into the deep tissue, whereas later subclones have more malignant capabilities that enable deeper invasion and metastasis. Hence if the ancestral trees represent stepwise selection, more invasive subclones should arise late on an ancestral tree (Figs. 1c, left). Saturation microdissection can directly test whether early and late subclones co-localize with superficial and invasive phenotypes, respectively.

Contrary to the expectations from the stepwise progression model, we found that most tumor sections contained multiple, vertically arranged and millimeter-sized subclones that shared invasive and superficial phenotypes. In 9 of 11 carcinomas, invasive phenotypes arose multiple times (multiclonal invasion) from both early and late branches, which suggests branching evolution rather than stepwise progression with invasive bottlenecks. Only 2 of 11 carcinomas harbored private driver mutations that indicate possible selection during growth. One of them (tumor D) had many features of stepwise progression but its dominant subclone arose early in its ancestral tree and not directly from neighboring superficial subclones. Therefore, while it is possible that more direct progenitor lesions were missed during sampling or may have been replaced during expansion of the dominant clone, the data do not support a late bottleneck to invasion in this tumor.

The observed dissociation of phenotypic and genotypic progression during tumor growth is consistent with near neutral evolution or Big Bang tumorigenesis ${ }^{11}$ where the founder cell already starts at a high fitness level and has the driver mutations sufficient for rapid growth (Fig 1B). Aligned with this scenario, most driver mutations in this and other studies ${ }^{9,18}$ were clonal, that is present in all sampled areas. The subclones were defined largely 
by passenger mutations that show little evidence of selection in cancers ${ }^{19-21}$, and unsurprisingly, the subclones mapped by saturation microdissections were present in both the invasive and non-invasive components without distinct boundaries between those components.

Genetic subclones with multiple phenotypes imply plasticity, where the same cell can adopt multiple phenotypes depending on its microenvironment ${ }^{16,22}$. Phenotypic plasticity allows for rapid growth because the founder cell and its progeny can readily adapt and have either invasive or superficial phenotypes depending on their locations within the tumor. Multiregional sampling of the same tumors showed preferentially conserved epigenomes as evidenced by preservation of unmethylated promoters and enhancers between opposite tumor sides ${ }^{10}$. Interestingly, phenotypic plasticity is already present in normal colon where differentiated cells share similar epigenomes and cell phenotype is determined by the microenvironment ${ }^{23}$.

Given a founder cell with the driver mutations and an epigenome sufficient for rapid growth and adaptation, invasion can occur very early and by multiple, essentially identical subclones. The ancestral trees inferred in this study likely reflect very early events in the first tumor gland because subclonal mutations that occur later would not be detectable by exome sequencing ${ }^{9,15}$. Consequently, the final locations of subclones within a tumor depend primarily on cell movement or mixing during early growth ${ }^{9}$, which in turn leads to the observed jigsaw-arranged subclone patterns that exhibit little correlation between physical and ancestral distances.

The vertically arranged subclone patterns can help explain why invasive and metastatic phenotypes arise early on ancestral trees. Indeed, with phenotypic plasticity, the initial direction of cell growth determines subclone phenotype because downwards (deep) growing subclones have greater physical access to stroma and vasculature than upwards (superficial) growing subclones. This in turn leads to star-shaped ancestral trees ${ }^{24}$ where invasion and metastasis can begin from the start of growth rather than being constrained by a bottleneck until superficial tumor growth is completed. The early ancestral branching of metastases is a common observation in human cancers ${ }^{18,25-28}$, and as corroborated by our findings, likely occurs very early when the primary tumor consists of less than one million cells ${ }^{29}$.

In summary, by merging ancestral, topographic, and phenotypic information from microdissected slides, we developed an experimental approach that provides direct insights into the growth of individual human tumors. 
Importantly, and in contrast to previous studies on this subject, our approach is not constrained by modeling assumptions that are hard to verify experimentally ${ }^{14,15}$. Although the studied tumors are more consistent with single neutral Big Bang expansions, there is considerable heterogeneity within and between tumors, which may reflect complex microenvironmental interactions or selection at scales smaller than the current millimeter resolution ${ }^{30}$. By increasing the number of micro-dissected spots and targeting private mutations with lower allelic frequencies, characterization of heterogeneity could be further refined and used to reconstruct how lethal human cancers start to grow. Indeed, mass screening programs have led to widespread overdiagnosis and overtreatment of small tumors ${ }^{31}$, and tackling these issues requires effective discrimination between indolent and lethal lesions at time of diagnosis. As such, the reconstruction of patient-specific ITH patterns from routine diagnostic materials as illustrated in this study may further enhance the delivery of effective personalized oncology. 


\section{ACKNOWLEDGEMENTS}

This work was supported in parts by grants from the Triangle Center for Evolutionary Medicine, National Institutes of Health (R00 CA207872, P30 CA014236, U54 CA217376, U2C CA233254, P01 CA91955, R01 CA170595, R01 CA185138, R01 CA140657), the National Science Foundation (DMS 1614838), CDMRP Breast Cancer Research Program Award (BC132057) and Arizona Biomedical Research Commission (ADHS18-198847).

\section{AUTHOR CONTRIBUTIONS}

DS and MDR designed the experiments, analyzed the data and wrote the manuscript. AH, TH, LMK, ICS, CCM, JRM, and ESH assisted with data analysis, interpretation, and manuscript editing.

\section{COMPETING INTERESTS}

The authors declare no competing interests. 


\section{METHODS}

Tumor samples. The 12 colorectal tumors (Table 1) were previously studied ${ }^{9}$ and collected as excess tissues in the course of routine clinical care. The studies were approved by the Institutional Review Board at the University of Southern California Keck School of Medicine.

Saturation microdissection and deep resequencing. Microdissection was performed using selective ultraviolet light fraction (SURF) as previously described ${ }^{32}$. Briefly, microscopic sections were placed on plastic slides, lightly stained, and small ink dots were placed directly over 2-5 tumor glands using a micromanipulator (Fig S5). Unprotected DNA was destroyed by 3-4 hours of short-wave ultraviolet light irradiation. The spots on the plastic slide were cut out and individually placed in a microfuge tube for DNA extraction (TE and Proteinase $\mathrm{K}, 60 \mathrm{C}$ for 4 hours, then $98^{\circ} \mathrm{C}$ for 10 minutes), using a pipette tip to remove the ink dot from the plastic slide. AMPure XP beads (Beckman Coulter) were added (1.2X) to extract the DNA. PCR (35-40 cycles) was directly performed on the dried beads, using custom AmpliSeq primers for tumor-specific single nucleotide variants (SNVs). The latter comprised both public (present in all samples) and private (present in strict subset of samples only) mutations as identified in a previous study ${ }^{9}$. Barcoded libraries were made (One-step, Qiagen) and run on MiSeq or NextSeq Illumina sequencers. Average coverage was $\sim 9,000 x$ with a minimum of 20 reads per spot required for the genotyping. The initial mutation calling threshold was a VAF of 0.05 . The complete data set (VAFs) is found in Supplemental Data.

Computer code. The analyses described below were all performed with the software $\mathrm{R}$ (version $3.5 .3, \mathrm{R}$ Foundation for Statistical Computing, Vienna, Austria). [NOTE TO REVIEWERS: code will be made available on the code sharing site GitHub in case of acceptance of the manuscript.]

Phylogenetic tree construction. Prior to ancestral reconstruction, two additional pre-processing steps were implemented for each tumor: (i) if a private mutation was found in only one spot and with a VAF $<6 \%$, it was interpreted as noise and classified as absent; (ii) if a mutation was present in more than $90 \%$ of all spots in the tumor, missingness of the mutation in the remaining spots was deemed a false negative finding and the mutation was classified as public. After pre-processing, the maximum parsimony trees were constructed using Nixon's ratchet algorithm ${ }^{33}$ as implemented in the phangorn package in $R$. Bootstrap sampling $(n=1,000)$ was performed to quantify tree building confidence. Trees were constructed under the principle of homoplasy avoidance by 
assuming that each mutation is acquired only once, and that SNVs are not selectively reversed during evolution (both events have a very low probability). Homoplasy avoidance was achieved as follows: (i) by removal of spots whose VAF spectrum indicated admixture of 2 or more subclones $(n=4)$; (ii) reclassification of false-positive and false negative calls $(n=12)$. See Supplemental Table 1 for details on homoplasy avoidance.

t-SNE plots. To visualize genotypic and phenotypic clustering properties of individual spots (Figs 3e, S2), we used t-distributed stochastic neighbor embedding, or t-SNE ${ }^{34}$, as implemented in the package Rtsne in R.

Normalized distance between adjacent subclones. This measure was computed to evaluate the genetic distance between spatially adjacent subclones (Fig 3g). For each tumor (except J, which only had one subclone), adjacent subclones were identified based on the mutation topographies (Figs 2C, 3, 4, S2). For each pair of adjacent subclones, the number of separating internal nodes was counted. A normalized metric was obtained by subtracting 1 and dividing the resulting integer by the maximum distance between any two tips on the tree.

Spatial vs. genetic distances. The pairwise spatial distances between spots on the same microscope sections were manually recorded. The genetic distance between spots was obtained, after processing (see above), as the $\mathrm{L}_{1}$-distance (Manhattan distance) between dichotomized VAF vectors. To quantify the correlations between genetic and spatial distances (Fig 3e), the Pearson correlation coefficients between the two measures were computed for each tumor. 


\begin{tabular}{|c|c|c|c|c|c|c|c|c|c|c|c|c|c|c|}
\hline ID & Type & $\begin{array}{l}\text { Size } \\
(\mathrm{cm})\end{array}$ & Stage & Slides & Spots & $\begin{array}{c}\text { Loci } \\
\text { assayed, n }\end{array}$ & $\begin{array}{c}\text { Mutations: } \\
\text { public/ } \\
\text { private }\end{array}$ & $\begin{array}{c}\text { Sub- } \\
\text { clones, } \\
\mathbf{n}\end{array}$ & $\begin{array}{c}\text { Clone } \\
\text { phenotypes }+ \\
\text { S/I/S-I }\end{array}$ & $\begin{array}{l}\text { Monophyle- } \\
\text { tic invasion }\end{array}$ & $\begin{array}{c}\text { Early } \\
\text { invasion }\end{array}$ & $\begin{array}{c}\text { Multiclo- } \\
\text { nal } \\
\text { invasion }\end{array}$ & $\begin{array}{l}\text { Invasion } \\
\text { events }\end{array}$ & $\begin{array}{c}\text { Early } \\
\text { mixing }+\dagger\end{array}$ \\
\hline $\mathrm{C}$ & CRC & 6.4 & S3 & 2 & 16 & 57 & $27 / 20$ & 9 & $8 / 0 / 0$ & Yes & Yes & No & 1 & No \\
\hline D & CRC & 2.0 & S1 & 2 & 33 & 57 & $23 / 27$ & 5 & $4 / 0 / 2$ & No & Yes & Yes & 2 & Yes \\
\hline$E$ & CRC & 6.1 & $\mathrm{~S} 1$ & 2 & 27 & 110 & $54 / 36$ & 6 & $2 / 2 / 2$ & No & Yes & Yes & 3 & Yes \\
\hline $\mathrm{F}$ & CRC & 1.8 & $\mathrm{~S} 1$ & 2 & 31 & 53 & $29 / 16$ & 5 & $1 / 1 / 3$ & No & Yes & Yes & 4 & Yes \\
\hline $\mathrm{H}$ & CRC & 4.0 & S4 & 2 & 39 & 30 & $12 / 10$ & 3 & $0 / 1 / 2$ & Yes & Yes & Yes & 3 & No \\
\hline$J$ & CRC & 5.0 & S3 & 2 & 27 & 28 & $17 / 0$ & 1 & $0 / 0 / 1$ & Yes & Yes & No & 1 & No \\
\hline $\mathrm{K}$ & $A d x$ & 6.0 & - & 2 & 29 & 41 & $11 / 25$ & 9 & $7 / 0 / 3^{*}$ & No* & Yes* & Yes* & $3^{*}$ & No \\
\hline$M$ & CRC & 3.0 & $\mathrm{~S} 2$ & 2 & 29 & 13 & $7 / 6$ & 7 & $0 / 2 / 5$ & Yes & Yes & Yes & 7 & Yes \\
\hline $\mathrm{R}$ & CRC & 3.5 & $\mathrm{~S} 1$ & 1 & 15 & 58 & $36 / 6$ & 3 & $0 / 1 / 2$ & Yes & Yes & Yes & 3 & Yes \\
\hline $\mathrm{T}$ & CRC & 5.7 & S3 & 2 & 26 & 35 & $22 / 5$ & 5 & $0 / 1 / 4$ & Yes & Yes & Yes & 5 & Yes \\
\hline U & CRC & 3.9 & $\mathrm{~S} 2$ & 2 & 23 & 29 & $25 / 3$ & 4 & $1 / 1 / 2$ & No & Yes & Yes & 3 & Yes \\
\hline W & CRC & 3.4 & $\mathrm{~S} 1$ & 2 & 30 & 68 & $59 / 9$ & 7 & $1 / 0 / 6$ & No & Yes & Yes & 6 & Yes \\
\hline \multicolumn{2}{|c|}{ Summary } & - & - & - & $\begin{array}{l}\text { Total: } \\
325\end{array}$ & $\begin{array}{l}\text { Total: } \\
579\end{array}$ & $\begin{array}{l}\text { Total: } \\
322 / 163\end{array}$ & $\begin{array}{l}\text { Median: } \\
5\end{array}$ & $\begin{array}{c}\text { Total: } \\
\text { 24/9/32 }\end{array}$ & $\begin{array}{c}\text { Total }^{* *:} \\
6 / 11\end{array}$ & $\begin{array}{c}\text { Total }^{* *:} \\
11 / 11\end{array}$ & $\begin{array}{c}\text { Total }^{* *}: \\
\text { 9/11 }\end{array}$ & $\begin{array}{c}\text { Median }^{* * *}: \\
3\end{array}$ & Total: 8/12 \\
\hline
\end{tabular}

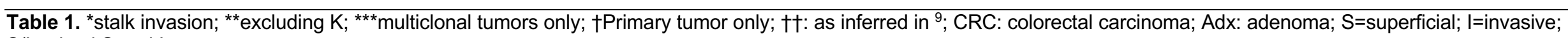
$\mathrm{S} / \mathrm{I}=$ mixed $\mathrm{S}$ and $\mathrm{I}$. 


\section{FIGURES}
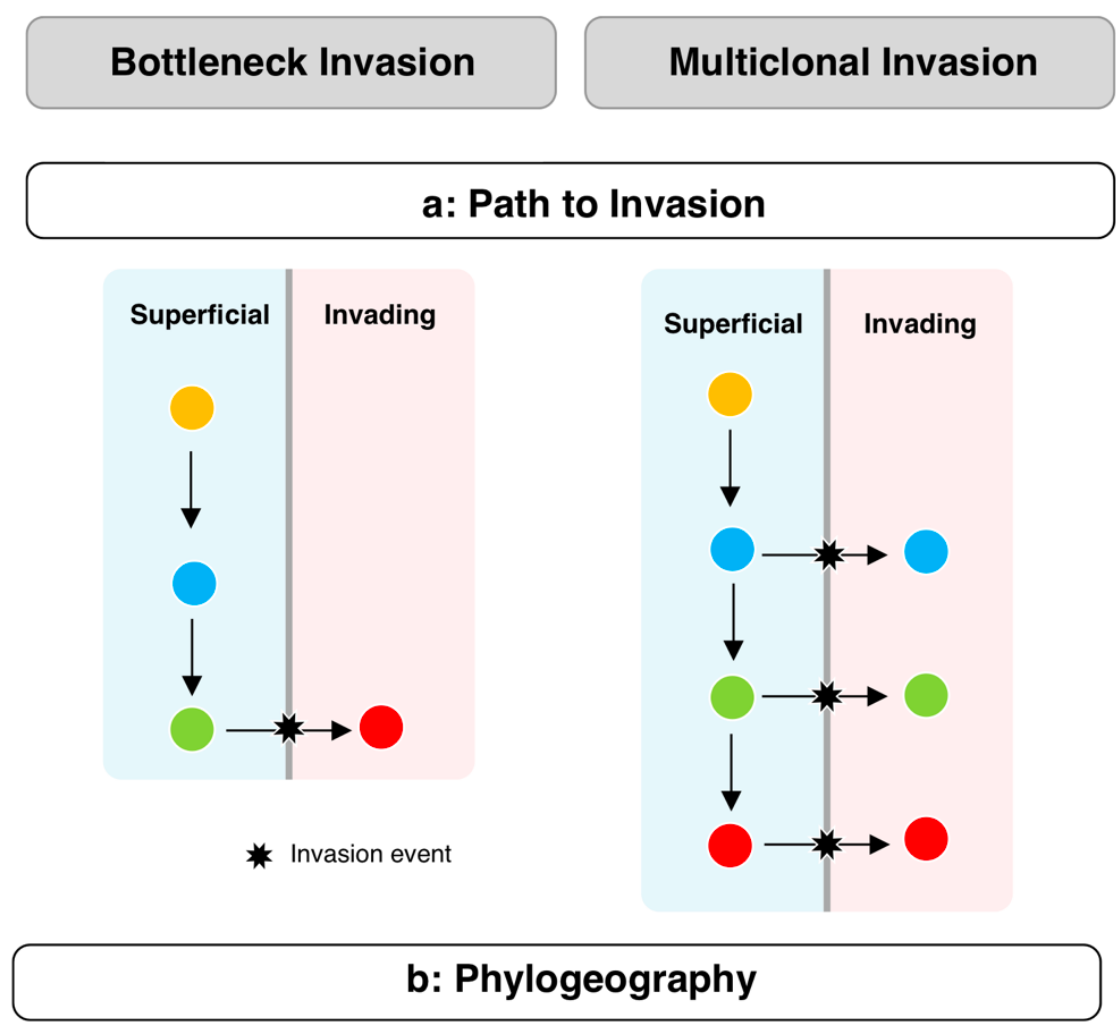

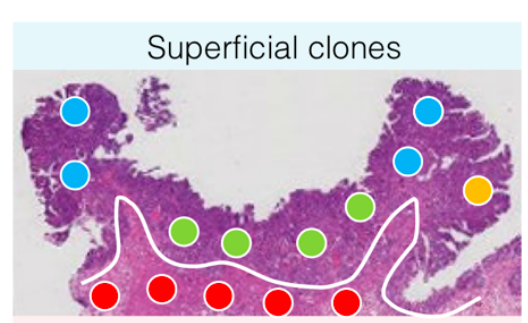

Invading clones

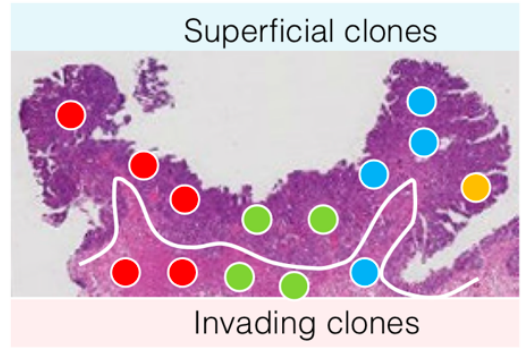

Invading clones

\section{c: Evolutionary Dynamics}
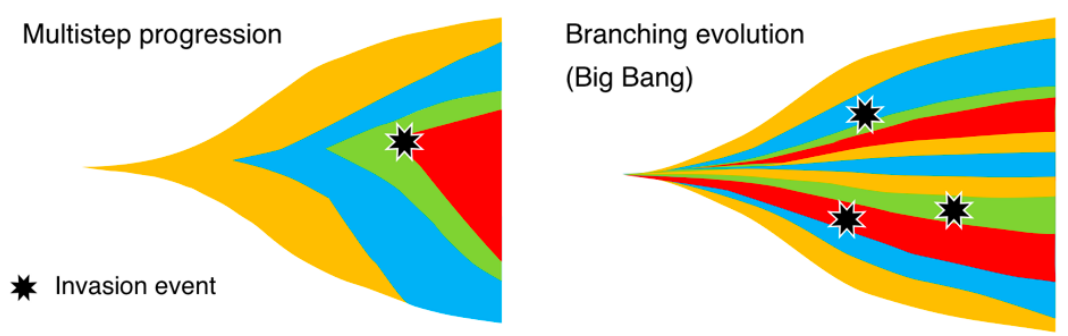

Figure 1. Bottleneck vs Multiclonal Invasion. Topographic and phenotypic subclone distributions generally differ between the bottleneck (left) and multiclonal invasion (right) models. (a) In the bottleneck model (left), the tumor evolves in superficial regions and invasion constitutes an evolutionary bottleneck where only the more advanced subclones eventually invade the stroma. In the multiclonal invasion scenario (right), co-existing subclones have a similar malignant potential to invade the stroma. (b) Left: if growth occurs through bottlenecks, subclones should be arranged horizontally with respect to phenotype, with a smaller number of more "advanced" subclones (red) with invasive phenotypes. Right: under multiclonal invasion, subclones have similar malignant potential; expansile growth occurs radially in all directions resulting in vertical subclones columns with both superficial and invasive phenotypes in the final tumor. (c) Left: the bottleneck model is compatible with the classic multi-step progression of cancer evolution whereby the invasive clone arises late (star). Right: the multiclonal invasion scenario is compatible with branching evolutionary models with multiple co-evolving subclones, such as predicted by the Big Bang model. 


\section{a) Tumor preparation}
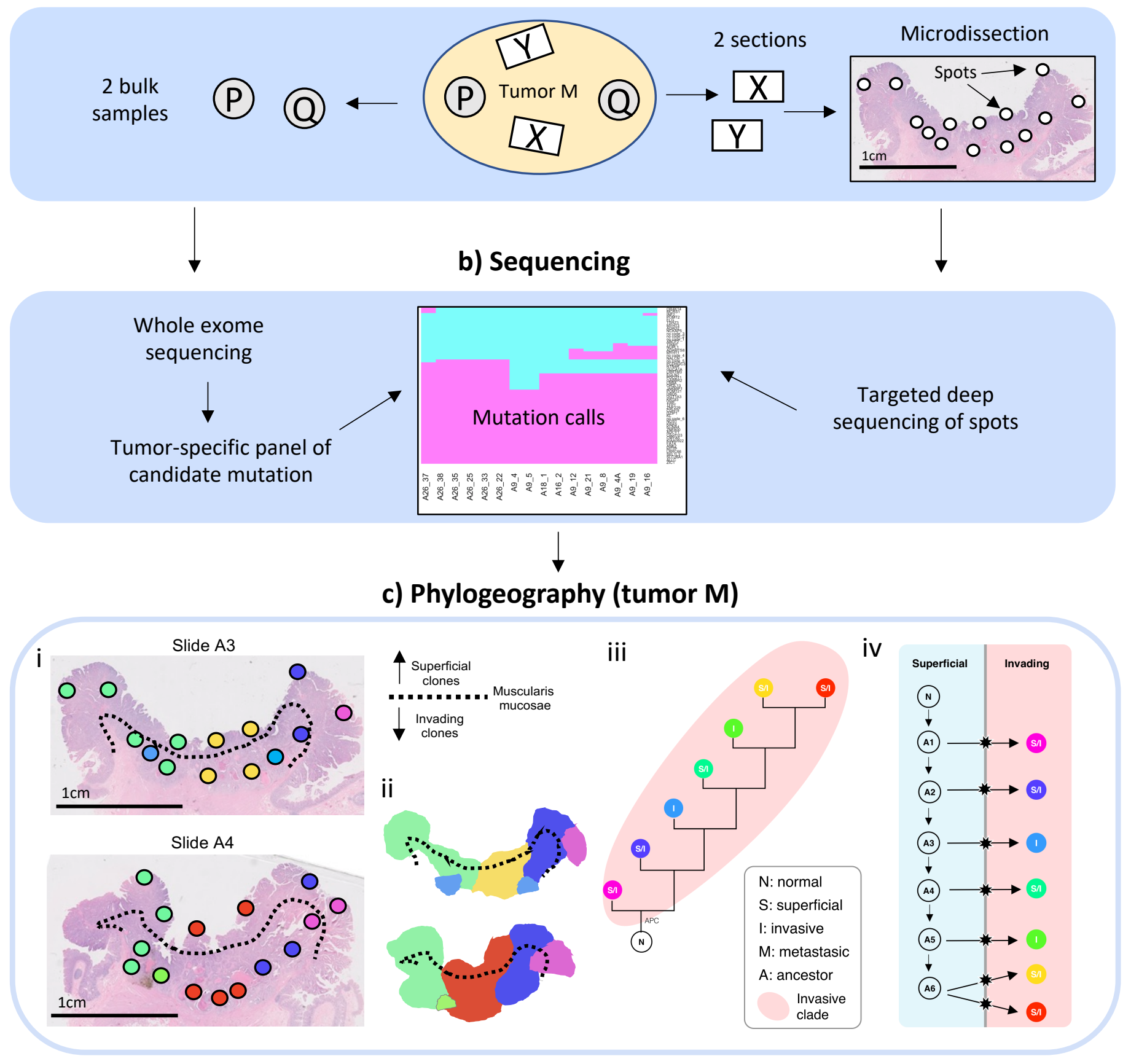

Figure 2. Study design. (a) From each tumor, two bulk samples from opposite sides were obtained ( $P, Q)$ and two microscope sections $(\mathrm{X}, \mathrm{Y})$ were collected during routine clinical work (arbitrary locations). From the microscopic sections, small spots (2-5 glands each) were microdissected using SURF technology. (b) After whole exome sequencing of the bulk samples $P$ and $Q$, tumor-specific mutation panels with $\sim 50$ candidate mutations were designed. For each spot from the sections $X$ and $Y$, targeted deep re-sequencing of the candidate mutations was performed. Because individual spots were clonal, mutations were called using a noise threshold for variant allele frequencies (VAF) at 5\%. (c) Tumor M. Spot genotypes were identified and localized on the sections, and sections were annotated for superficial and invasive regions (i). Invasion was defined as migration below the muscularis mucosae (dotted line). Contiguous clone maps were derived from the spot topographies (ii). Maximum parsimony algorithms were used to reconstruct phylogenetic trees for the subclones (iii). The ancestry of invasive subclones was reconstructed under the assumption that cells can migrate from superficial to invasive regions, but not vice-versa (iv). 

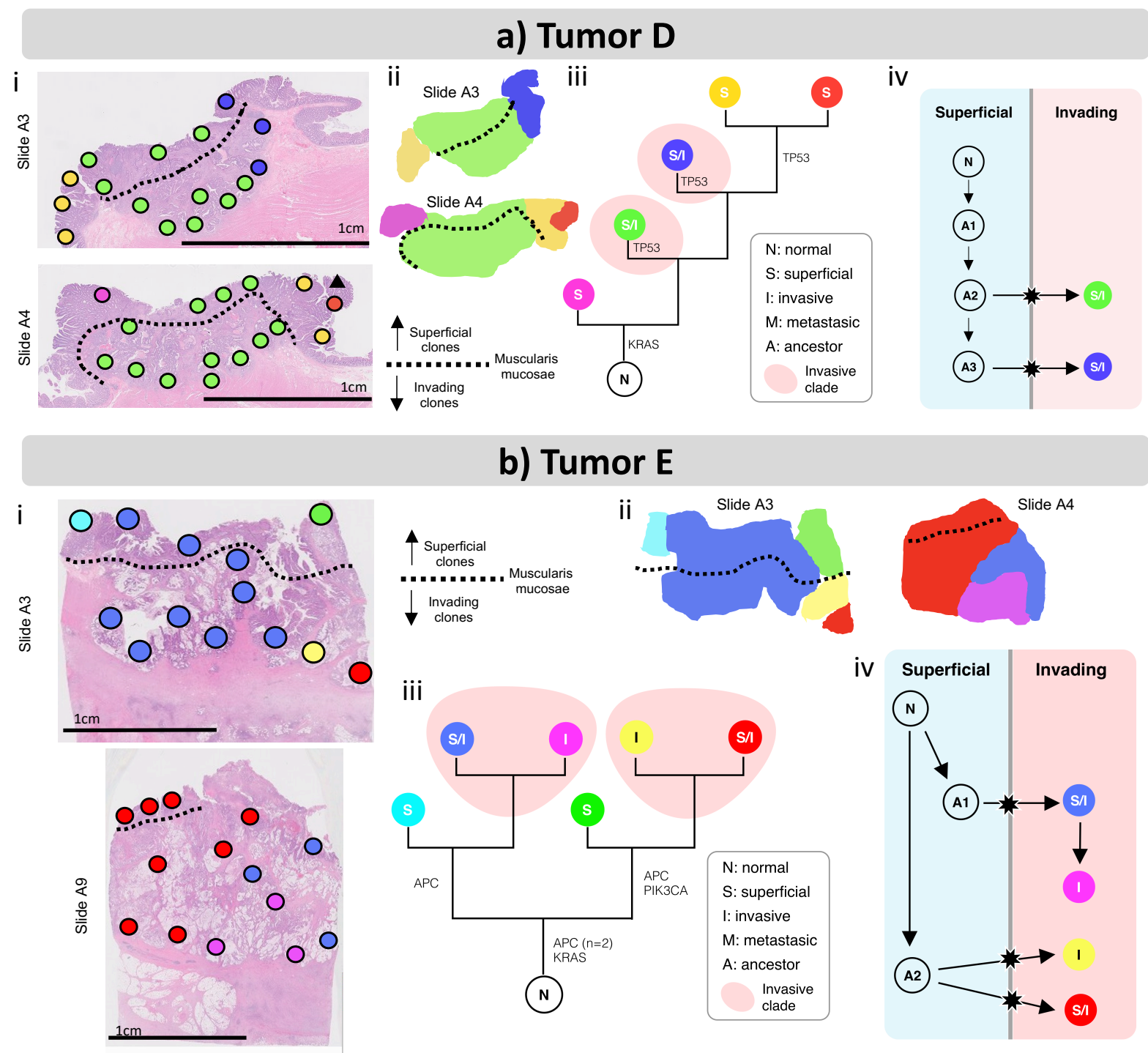

\section{b) Tumor $E$}
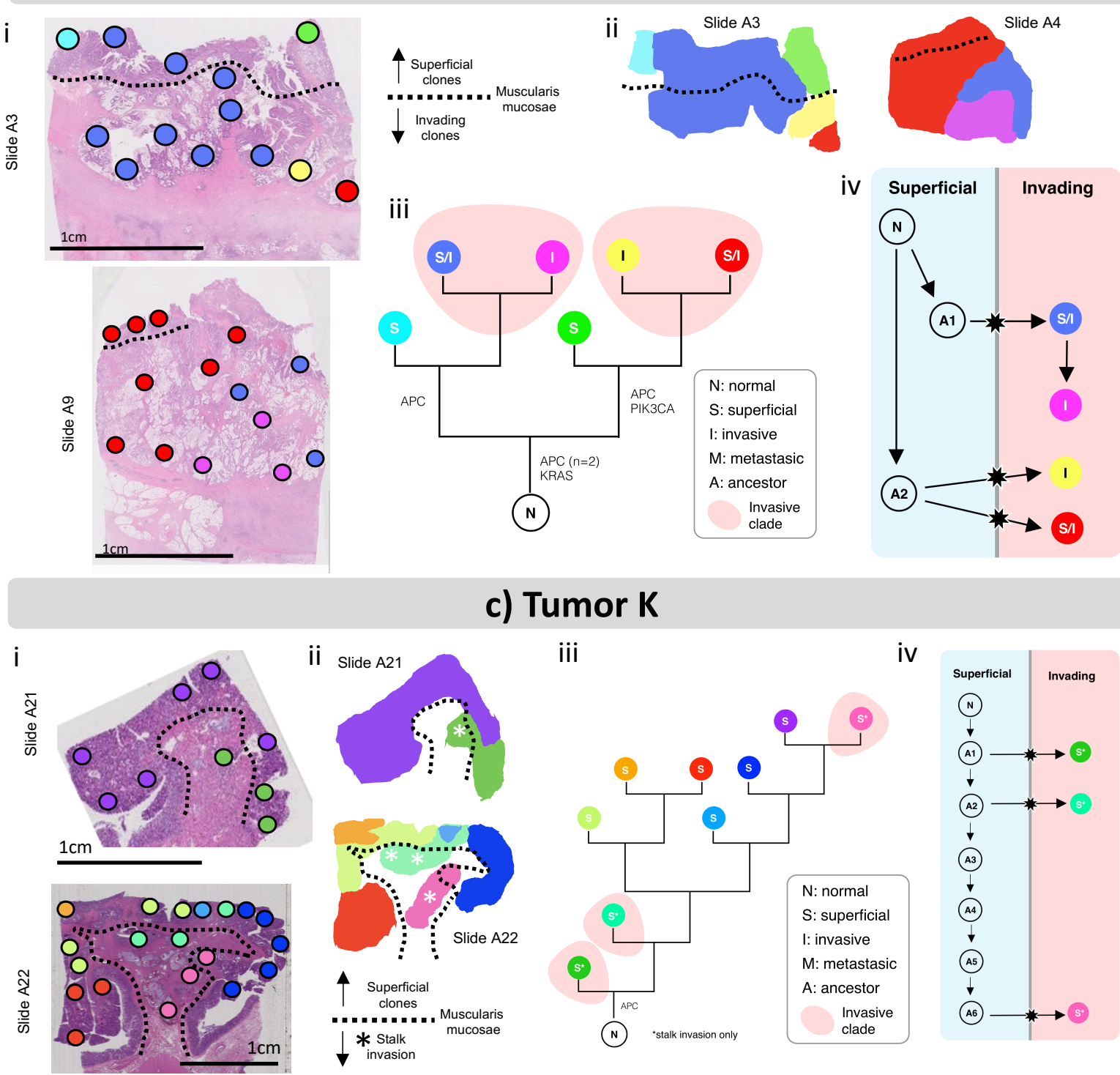

\section{) Tumor K}

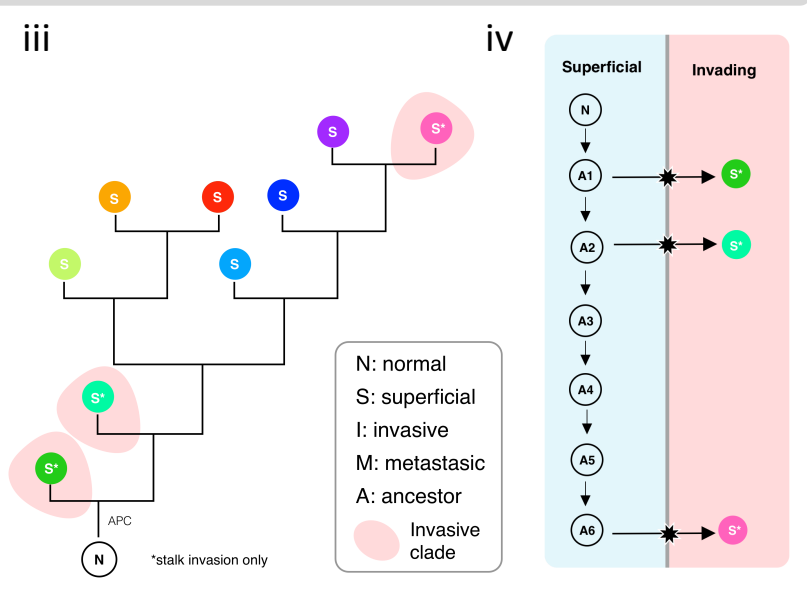

Figure 3. Phylogeographies of two carcinomas $(\mathbf{a}, \mathbf{b})$ and one adenoma (c). Spot genotypes were identified and localized on the sections, and sections were annotated for superficial and invasive regions (i). Invasion was defined as migration below the muscularis mucosae (dotted line). The sole adenoma (tumor K) had focal stalk invasion, which may represent the start of deeper invasion. Contiguous clone maps were derived from the spot topographies, revealing vertically arranged subclones that share superficial and invasive phenotypes (ii). Maximum parsimony algorithms were used to reconstruct phylogenetic trees for the subclones (iii). The ancestry of invasive subclones was reconstructed under the assumption that cells migrate from superficial to invasive regions, but not vice-versa (iv). 

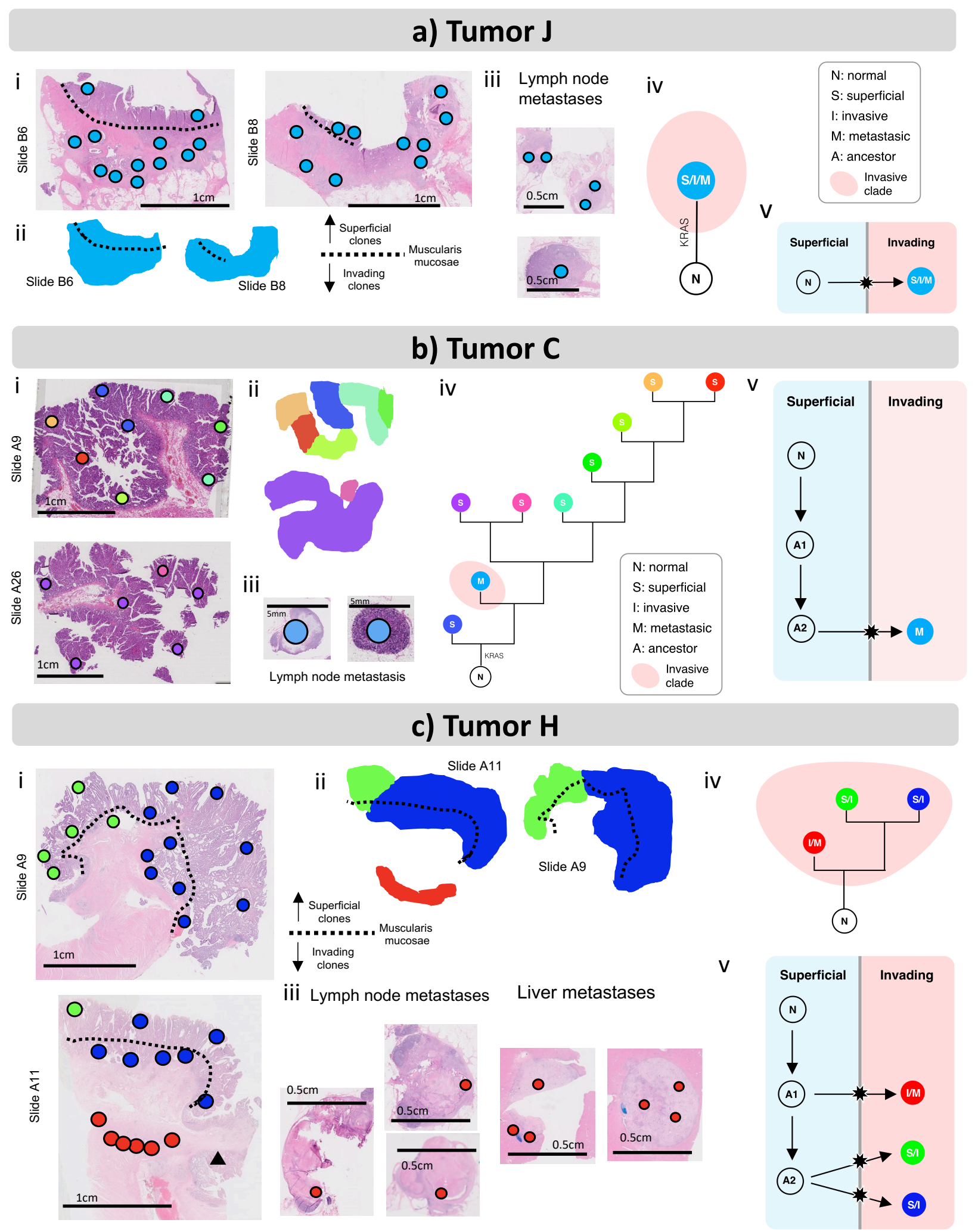

Figure 4. Microscopic metastases and early branching in three tumors (a,b,c). Spot genotypes were identified and localized on the sections, and sections were annotated for superficial and invasive regions (i). In tumor $\mathrm{J}$, no private mutations were identified in primary or metastases. In tumor $\mathrm{H}$, a deeply invasive ribbon of cells (red, slide A11) shared the genotype of the metastases, yet did not share the private mutations of the overlying superficial portions of the primary. Contiguous clone maps were derived from the spot topographies (ii). Additional spots in the lymph nodes and liver metastases (tumor $\mathrm{H}$ only) were microdissected (iii). Maximum parsimony algorithms were used to reconstruct phylogenetic trees for the subclones (iv). In tumor C, the two lymph node spots shared genotypes and branched early before the divergence of the two halves. The metastatic genotype was not found in the primary tumor. The ancestry of invasive subclones was reconstructed under the assumption that cells migrate from superficial to invasive regions, but not vice-versa ( $v$ ). 
a

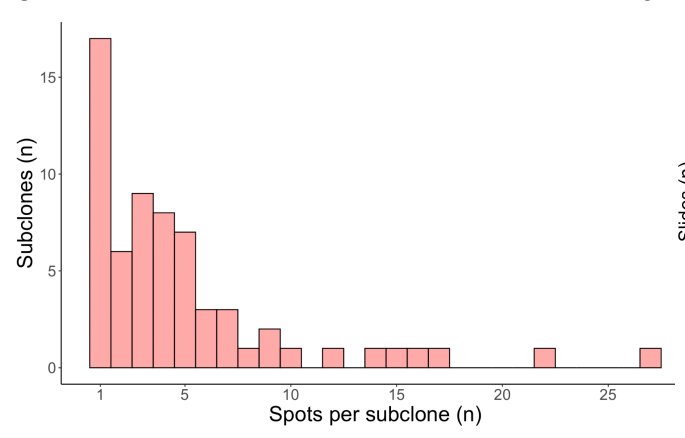

b

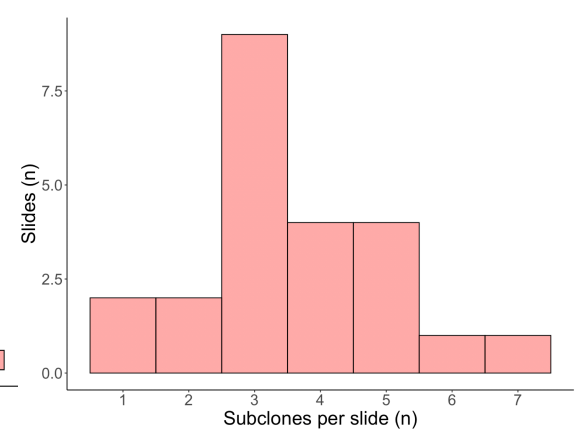

C

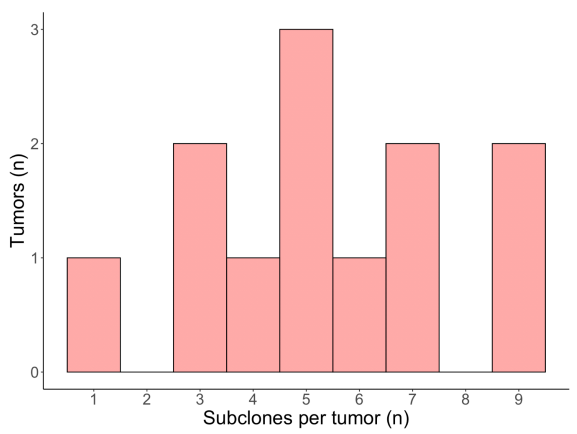

d

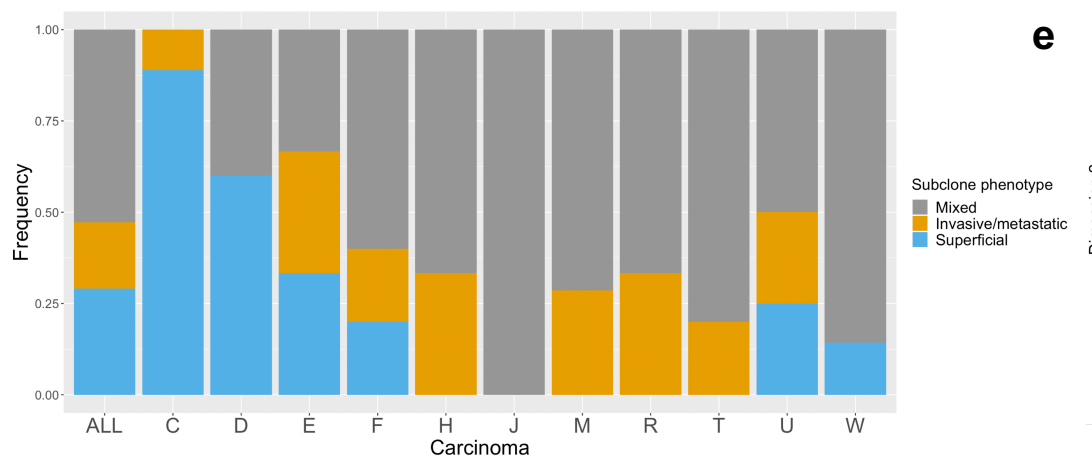

f

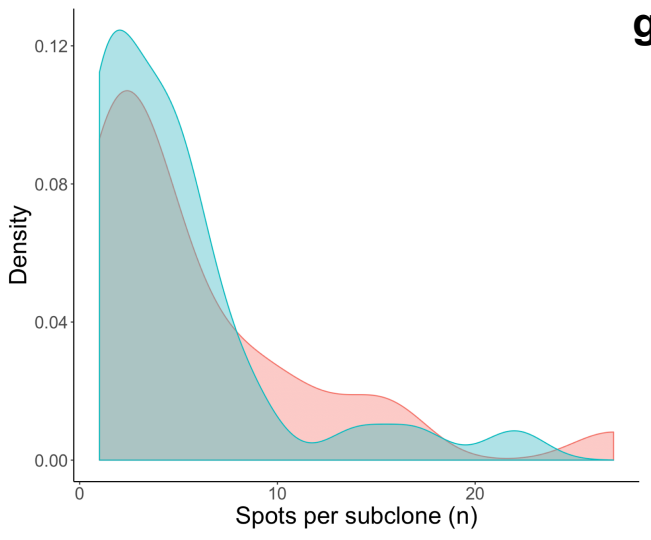

g

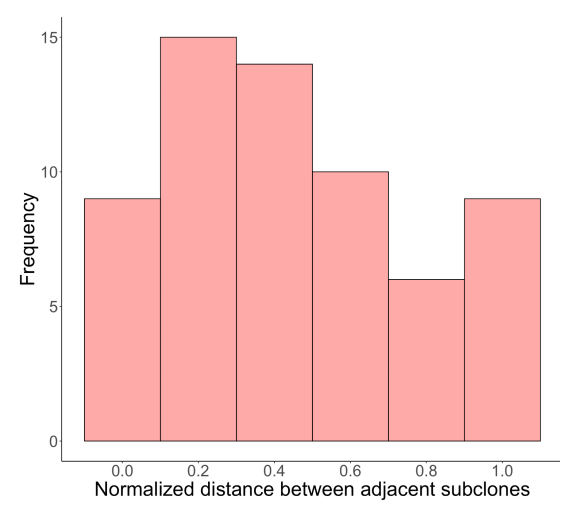

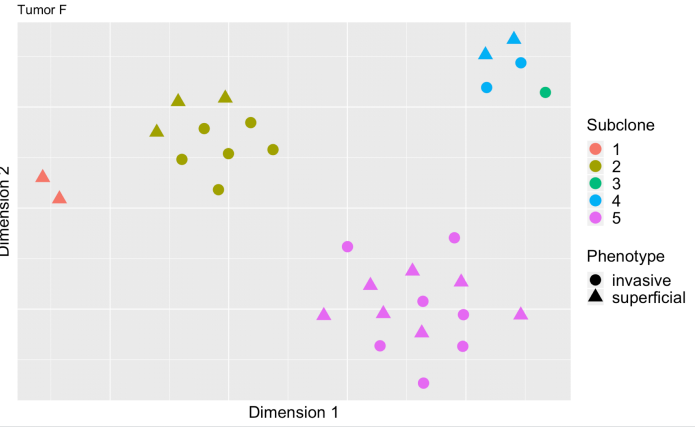

h

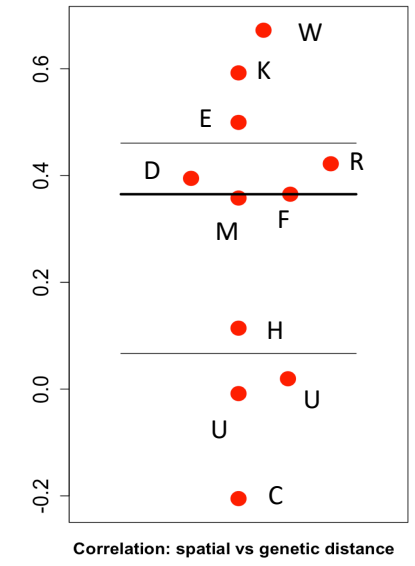

Figure 5. Summary statistics. (a) Across all 12 tumors, the number of spots per subclone ranged from 1 to 27 (median: 3.5 ). (b) Across all 23 microscopic tissue slides, the number of subclones per slide ranged from 1 to 7 (median: 3). (c) The number of subclones per tumor ranged from 1 to 9 (median: 5). (d) With the exception of tumor C, all tumors had subclones of mixed phenotype, i.e., the same genotype was found in superficial and invasive/metastatic spots of the tumor. (e) Nonlinear embedding (t-SNE) of the high-dimensional sequencing data for tumor $\mathrm{F}$ illustrates that spots cluster by genotype (colors) but not by phenotype (shapes). t-SNE plots for all tumors in Fig. S3. (f) Early and late subclones (as measured by relative distance from the root of the ancestral tree, see Fig S4) had similar subclone distributions. (g) The normalized phylogenetic distance (number of separating internal nodes) between physically adjacent subclones was broadly distributed. (h) The tumor-specific Pearson correlation between genetic and phylogenetic distances ranged from -0.2 to 0.8 (median: 0.37). 


\section{REFERENCES}

1 Davis, A., Gao, R. \& Navin, N. Tumor evolution: Linear, branching, neutral or punctuated? Biochimica et Biophysica Acta (BBA)-Reviews on Cancer 1867, 151-161 (2017).

Greaves, M. \& Maley, C. C. Clonal evolution in cancer. Nature 481, 306-313, doi:10.1038/nature10762 (2012).

Burrell, R. A., McGranahan, N., Bartek, J. \& Swanton, C. The causes and consequences of genetic heterogeneity in cancer evolution. Nature 501, 338-345, doi:10.1038/nature12625 (2013).

Gallaher, J. A., Enriquez-Navas, P. M., Luddy, K. A., Gatenby, R. A. \& Anderson, A. R. Spatial heterogeneity and evolutionary dynamics modulate time to recurrence in continuous and adaptive cancer therapies. Cancer research 78, 2127-2139 (2018).

Maley, C. C. et al. Classifying the evolutionary and ecological features of neoplasms. Nature Reviews Cancer 17, 605, doi:10.1038/nrc.2017.69 (2017).

Stanta, G. \& Bonin, S. Overview on Clinical Relevance of Intra-Tumor Heterogeneity. Front Med (Lausanne) 5, 85, doi:10.3389/fmed.2018.00085 (2018).

Fearon, E. R. \& Vogelstein, B. A genetic model for colorectal tumorigenesis. cell 61, 759-767 (1990). Future. Cell 168, 613-628, doi:10.1016/j.cell.2017.01.018 (2017). (2018).

Ryser, M. D., Yu, M., Grady, W., Siegmund, K. \& Shibata, D. Epigenetic Heterogeneity in Human Colorectal Tumors Reveals Preferential Conservation And Evidence of Immune Surveillance. Sci Rep 8, 17292, doi:10.1038/s41598-018-35621-y (2018).

11 Sottoriva, A. et al. A Big Bang model of human colorectal tumor growth. Nat Genet 47, 209-216, doi:10.1038/ng.3214 (2015).

12 Casasent, A. K. et al. Multiclonal Invasion in Breast Tumors Identified by Topographic Single Cell Sequencing. Cell 172, 205-217 e212, doi:10.1016/j.cell.2017.12.007 (2018).

13 Humphries, A. \& Wright, N. A. Colonic crypt organization and tumorigenesis. Nat Rev Cancer 8, 415424, doi:10.1038/nrc2392 (2008).

14 McDonald, T. O., Chakrabarti, S. \& Michor, F. Currently available bulk sequencing data do not necessarily support a model of neutral tumor evolution. Nature Genetics 50, 1620-1623, doi:10.1038/s41588-018-0217-6 (2018).

15 Williams, M. J. et al. Quantification of subclonal selection in cancer from bulk sequencing data. Nat Genet 50, 895-903, doi:10.1038/s41588-018-0128-6 (2018).

16 Meacham, C. E. \& Morrison, S. J. Tumour heterogeneity and cancer cell plasticity. Nature 501, 328337, doi:10.1038/nature12624 (2013).

17 Sun, R. et al. Between-region genetic divergence reflects the mode and tempo of tumor evolution. Nat Genet 49, 1015-1024, doi:10.1038/ng.3891 (2017).

18 Reiter, J. G. et al. Minimal functional driver gene heterogeneity among untreated metastases. Science 361, 1033-1037, doi:10.1126/science.aat7171 (2018).

19 Martincorena, I. et al. Universal Patterns of Selection in Cancer and Somatic Tissues. Cell 171, 10291041 e1021, doi:10.1016/j.cell.2017.09.042 (2017).

20 Ling, S. et al. Extremely high genetic diversity in a single tumor points to prevalence of non-Darwinian cell evolution. Proc Natl Acad Sci U S A 112, E6496-6505, doi:10.1073/pnas.1519556112 (2015).

21 Williams, M. J., Werner, B., Barnes, C. P., Graham, T. A. \& Sottoriva, A. Identification of neutral tumor evolution across cancer types. Nat Genet 48, 238-244, doi:10.1038/ng.3489 (2016). Nature 506, 511-515, doi:10.1038/nature12903 (2014).

24 Humphries, A. et al. Lineage tracing reveals multipotent stem cells maintain human adenomas and the pattern of clonal expansion in tumor evolution. Proceedings of the National Academy of Sciences 110, E2490-E2499 (2013). 
25 Kim, T. M. et al. Subclonal Genomic Architectures of Primary and Metastatic Colorectal Cancer Based on Intratumoral Genetic Heterogeneity. Clin Cancer Res 21, 4461-4472, doi:10.1158/1078-0432.CCR14-2413 (2015).

26 Turajlic, S. \& Swanton, C. Metastasis as an evolutionary process. Science 352, 169-175, doi:10.1126/science.aaf2784 (2016).

27 Ulintz, P. J., Greenson, J. K., Wu, R., Fearon, E. R. \& Hardiman, K. M. Lymph Node Metastases in Colon Cancer Are Polyclonal. Clin Cancer Res 24, 2214-2224, doi:10.1158/1078-0432.CCR-17-1425 (2018).

28 Zhao, Z. M. et al. Early and multiple origins of metastatic lineages within primary tumors. Proc Natl Acad Sci U S A 113, 2140-2145, doi:10.1073/pnas.1525677113 (2016).

$29 \mathrm{Hu}$, Z. et al. Quantitative evidence for early metastatic seeding in colorectal cancer. Nat Genet 51, 1113-1122, doi:10.1038/s41588-019-0423-x (2019).

30 Maley, C. C. et al. Classifying the evolutionary and ecological features of neoplasms. Nat Rev Cancer 17, 605-619, doi:10.1038/nrc.2017.69 (2017).

31 Welch, H. G. \& Black, W. C. Overdiagnosis in cancer. Journal of the National Cancer Institute 102, 605$613(2010)$.

32 Shibata, D. et al. Specific genetic analysis of microscopic tissue after selective ultraviolet radiation fractionation and the polymerase chain reaction. Am J Pathol 141, 539-543 (1992).

33 Nixon, K. C. The parsimony ratchet, a new method for rapid parsimony analysis. Cladistics 15, 407-414 (1999).

34 Maaten, L. v. d. \& Hinton, G. Visualizing data using t-SNE. Journal of machine learning research $\mathbf{9}$, 2579-2605 (2008). 